\title{
Effects of first-order stress gradients in an ice sheet evaluated by a three-dimensional thermomechanical coupled model
}

\author{
Fuyuki SAITO, ${ }^{1}$ Ayako ABE-OUCHI, ${ }^{1}$ Heinz BLATTER ${ }^{2}$ \\ ${ }^{1}$ Centre for Climate System Research, The University of Tokyo, 4-6-1 Komaba, Meguro-ku, Tokyo 153-8904, Japan \\ E-mail:fuyuki@ccsr.u-tokyo.ac.jp \\ ${ }^{2}$ Institute for Climate Research, Eidgenössische Technische Hochschule, Winterthurerstrasse 190, CH-8057 Zürich, Switzerland
}

\begin{abstract}
A three-dimensional thermomechanically coupled ice-sheet model including calculation of the first-order stress gradients is developed to perform numerical studies on the effects of stress components neglected in the zeroth-order shallow-ice approximation. Steady-state solutions are obtained with both a shallow-ice and a first-order icesheet model for several idealistic, radially symmetric ice-sheet configurations. The results show that the effects of the normal deviatoric-stress gradients on ice thickness are generally small, but the influence on basal temperatures and age profiles at an ice divide is significant.
\end{abstract}

\section{INTRODUCTION}

There are several three-dimensional numerical ice-sheet models with thermodynamical coupling (Huybrechts, 1990; Ritz and others, 1997; Budd and others, 1998; Greve and others, 1998; Payne, 1999; Marshall and others, 2000). All of them are based on the shallow-ice approximation (SIA), which assumes that driving stress balances basal shear stress and also that the horizontal gradient of longitudinal deviatoric stress (not deviatoric stress itself) is negligibly small. This is also called zeroth-order approximation (Colinge and Blatter, 1998; Pattyn, 2000). SIA is employed mainly for saving computation time and easy development of numerical models. It captures general features of large "grounded" ice sheets and can represent present ice sheets well. However, this approximation breaks down near ice divides, in narrow regions of ice streams and near margin and shelf-sheet transition zones (e.g. Abe-Ouchi, 1993; Blatter, 1995).

The inclusion of horizontal normal (longitudinal) deviatoric stress and horizontal shear stress in the force-balance equations is called first-order approximation (Colinge and Blatter, 1998), or considered as an incomplete second-order approximation (Baral and others, 2001), which is hereafter referred to as first-order approximation (FOA). There have been several two-dimensional numerical ice-sheet models including the first- (or second-)order mechanics (DahlJensen, 1989a; Blatter, 1995; Colinge and Blatter, 1998; Pattyn 2000). Dahl-Jensen (1989a) demonstrated that longitudinal deviatoric stress has the same order of magnitude as vertical shear stress for plane flow along the flowline of an ice sheet, and that neither of them can be neglected. She also showed that inclusion of longitudinal deviatoric stress yields good ice-divide solutions. Blatter (1995) presented an efficient algorithm for three-dimensional calculation of the first-order mechanics and applied it to an ideal isothermal ice sheet. Pattyn (1996, 2000) and Pattyn and Decleir (1998) applied a two-dimensional full stress model with thermomechanical coupling to Shirase drainage basin, Dronning Maud Land, Antarctica, including Dome Fuji. They concluded that, among other things, changes in the dynamic behaviour of the coastal ice stream (Shirase Glacier) hardly influence the age-depth profile at Dome Fuji.

Hitherto, there has been no three-dimensional model with thermomechanical coupling that includes the computation of the first-order stress field. In this work, such a model is presented and is applied to a simple, radially symmetric geometry in order to investigate the effect of the firstorder stress terms in comparison with a shallow-ice model. Comparisons are made for steady-state shape (thickness distribution), temperature distribution and vertical profiles of age at an ice divide.

An ice divide or dome is a special place for deep core drilling. A divide is the region where vertical shear stress is almost zero, and horizontal normal stress dominates, and where flow characteristics cannot be expressed by the SIA. Interpretation of core data requires the depth-age relation, and numerical models are useful for this purpose. The age of ice is a function of flow, which changes through time in response to climatic forcing such as glacial/interglacial cycles. Dating by numerical models is therefore expected to be effective.

Ice fabrics and their mechanical properties are also provided by deep core drilling (Azuma and others, 1999; Hondoh and others, 1999; Miyamoto and others, 1999). Different fabrics cause different characteristics of deformation, and, conversely, fabrics depend much on the deformation and stress history. This is thought to influence the dating of an ice core by a flow model of the ice-sheet dynamics. Azuma (1994) and Goto-Azuma (1996) suggest a flow law for anisotropic icesheet ice, expressed in terms of the strain-rate, stress and geometric factor tensors. They demonstrate good agreement with observations and experimental results. Miyamoto and others (1999) performed mechanical tests on samples of the Greenland Icecore Project (GRIP) ice core. They found that the ice-flow enhancement factor against vertical shear 
rates shows a gradual increase with depth, becoming $>20$ times larger than for isotropic ice at about $2000 \mathrm{~m}$ depth. Because of their locations, interpretation of such fabric profiles requires higher-order stress contributions, which are not explicitly incorporated in the SIA model.

In the present paper, we focus on the divide and the effect of the horizontal longitudinal deviatoric stress. The horizontal shear stress is not discussed. This is the first attempt at developing the three-dimensional problem, and just the first application of higher-order three-dimensional ice-sheet models.

\section{MODEL DESGRIPTION}

The zeroth-order (SIA) ice-sheet model used in this study largely corresponds to the models described in detail in Payne and others (2000); the model in the FOA deviates from these models mainly in the computation of the flow field and the strain heating. The models contain two prognostic equations for the temperature and the surface, which depend on time $t$, and steady-state equations for stress and flow fields. The horizontal spatial variables are $x$ and $y$ and the vertical variable is $z$; the ice thickness $H=H(x, y)=h(x, y)-b(x, y)$, where $h$ and $b$ are the ice surface and base, respectively. In this section, only the differences between the FOA and the SIA are presented. The SIA models used in the European Ice-Sheet Modelling Initiative (EISMINT) model intercomparison are described in Payne and others (2000); a comprehensive description of the SIA is given in Greve (1997). The FOA model in the present work is based on Blatter (1995) and Colinge and Blatter (1998). It differs from the SIA models mainly in the computation of the velocity field and the strain heating.

The first-order force balance (Huybrechts, 1992; AbeOuchi, 1993; Blatter, 1995) is:

$$
\begin{aligned}
& 2 \frac{\partial \sigma_{x x}^{\prime}}{\partial x}+\frac{\partial \sigma_{y y}^{\prime}}{\partial x}+\frac{\partial \sigma_{x y}}{\partial y}-\rho_{\mathrm{I}} g \frac{\partial h}{\partial x}+\frac{\partial \sigma_{x z}}{\partial z}=0, \\
& 2 \frac{\partial \sigma_{y y}^{\prime}}{\partial y}+\frac{\partial \sigma_{x x}^{\prime}}{\partial y}+\frac{\partial \sigma_{x y}}{\partial x}-\rho_{\mathrm{I}} g \frac{\partial h}{\partial y}+\frac{\partial \sigma_{y z}}{\partial z}=0,
\end{aligned}
$$

where $\sigma_{x x}^{\prime}$ denotes the normal deviatoric-stress components in the corresponding directions, $\sigma_{i j}$ denotes the corresponding shear-stress components, and $\rho_{\mathrm{I}}$ and $g$ are the density of ice and the acceleration of gravity, respectively. The FOA force balance contains not only the gradients of vertical shear-stress components $\left(\sigma_{x z}, \sigma_{y z}\right)$, but also the normal deviatoric-stress and horizontal shear-stress components $\left(\sigma_{x x}^{\prime}, \sigma_{y y}^{\prime}, \sigma_{x y}\right)$.

The first-order constitutive equations (Glen's flow law) are:

$$
\begin{aligned}
\frac{\partial u}{\partial x} & =m A(T) \tau_{\mathrm{e}}^{n-1} \sigma_{x x}^{\prime}, \\
\frac{\partial v}{\partial y} & =m A(T) \tau_{\mathrm{e}}^{n-1} \sigma_{y y}^{\prime}, \\
\frac{1}{2} \frac{\partial u}{\partial z} & =m A(T) \tau_{\mathrm{e}}^{n-1} \sigma_{x z}, \\
\frac{1}{2} \frac{\partial v}{\partial z} & =m A(T) \tau_{\mathrm{e}}^{n-1} \sigma_{y z}, \\
\frac{1}{2}\left(\frac{\partial u}{\partial x}+\frac{\partial v}{\partial y}\right) & =m A(T) \tau_{\mathrm{e}}^{n-1} \sigma_{x y},
\end{aligned}
$$

where $u, v$ are the horizontal velocity components in the $x$ and $y$ directions, respectively, $m$ is an enhancement factor,
$T$ is temperature and $A$ is a temperature-dependent rate factor (Blatter, 1995). The horizontal gradients of vertical velocity component are neglected in Equations (2c) and (2d) because they are of the second order.

The second invariant of the deviatoric-stress tensor, $\tau_{\mathrm{e}}$, in the first-order approximation (Huybrechts, 1992) is:

$$
\begin{aligned}
\tau_{\mathrm{e}}^{2}=\frac{1}{2} \operatorname{tr}[\sigma \cdot \sigma]=\left(\sigma_{x x}^{\prime}\right)^{2} & +\left(\sigma_{y y}^{\prime}\right)^{2}+\sigma_{x x}^{\prime} \sigma_{y y}^{\prime}+\left(\sigma_{x y}\right)^{2} \\
& +\left(\sigma_{y z}\right)^{2}+\left(\sigma_{z x}\right)^{2}
\end{aligned}
$$

Velocity fields are calculated with surface and bedrock topography by the method of Blatter (1995) with Equations $(1-3)$. Then the local horizontal mass flux is obtained by vertical integration of the velocity fields, from which the evolution of the local ice thickness is determined.

The computation of the transient temperature field follows the method of the EISMINT models and considers horizontal and vertical ice advection but only vertical heat diffusion,

$$
\frac{\partial T}{\partial t}=\frac{k_{\mathrm{I}}}{\rho_{\mathrm{I}} c_{\mathrm{p}}} \Delta T-(\vec{v} \cdot \nabla) T+\frac{\Phi}{\rho_{\mathrm{I}} c_{\mathrm{p}}},
$$

where $c_{\mathrm{p}}$ and $k_{\mathrm{I}}$ are the specific heat capacity and the thermal diffusivity of ice.

The first-order strain-heating term $(\Phi)$ is:

$$
\begin{aligned}
\Phi=\frac{\partial u}{\partial z} \sigma_{x z} & +\frac{\partial v}{\partial z} \sigma_{y z}+\left(2 \frac{\partial u}{\partial x}+\frac{\partial v}{\partial y}\right) \sigma_{x x}^{\prime} \\
& +\left(2 \frac{\partial v}{\partial y}+\frac{\partial u}{\partial x}\right) \sigma_{y y}^{\prime}+\left(\frac{\partial u}{\partial y}+\frac{\partial v}{\partial x}\right) \sigma_{x y} .
\end{aligned}
$$

In the SIA, only the first two terms on the righthand side of Equation (5) are included.

The energy equation (4) is solved with prescribed surface temperature and geothermal heat flux $(\Gamma)$ at the bottom boundary,

$$
\begin{gathered}
T(z=h)=T_{\mathrm{s}}(x, y, t), \\
\left\{\begin{array}{c}
\left.\frac{\partial T}{\partial z}\right|_{z=b}=-\frac{\Gamma}{k_{\mathrm{I}}} \quad \text { if no melting, } \\
T(z=b)=T_{\mathrm{pm}} \quad \text { if melting, } 2
\end{array}\right.
\end{gathered}
$$

where $T_{\mathrm{pm}}$ is pressure-melting-point temperature.

The ice-stiffness (viscosity) coefficient, $A=A(T)$, strongly depends on ice temperature, and velocity fields and temperature are coupled through this factor. The factor $A$ is incorporated using an Arrhenius relation as follows:

$$
A\left(T^{\prime}\right)=a \exp \left(-\frac{Q}{R T^{\prime}}\right),
$$

where $T^{\prime}$ is absolute temperature corrected for the dependence of melting point on pressure (Paterson, 1994). Parameters $a$ and $Q$ are given by the following relations (e.g. Huybrechts, 1992; Payne and others, 2000):

$$
\begin{aligned}
& \left.\begin{array}{l}
a=3.61 \times 10^{-13} \mathrm{~Pa}^{-3} \mathrm{~s}^{-1} \\
\begin{array}{r}
=1.73 \times 10^{3} \mathrm{~J} \mathrm{~mol}^{-1}
\end{array}
\end{array}\right\} \quad \text { if } T^{\prime}<263.15 \mathrm{~K} \\
& \left.\begin{array}{rl}
a & =6.0 \times 10^{4} \mathrm{~Pa}^{-3} \mathrm{~s}^{-1} \\
Q & =13.9 \times 10^{4} \mathrm{~J} \mathrm{~mol}^{-1}
\end{array}\right\} \quad \text { if } T^{\prime} \geq 263.15 \mathrm{~K} .
\end{aligned}
$$

The computation of all other quantities corresponds to that in the SIA models. 


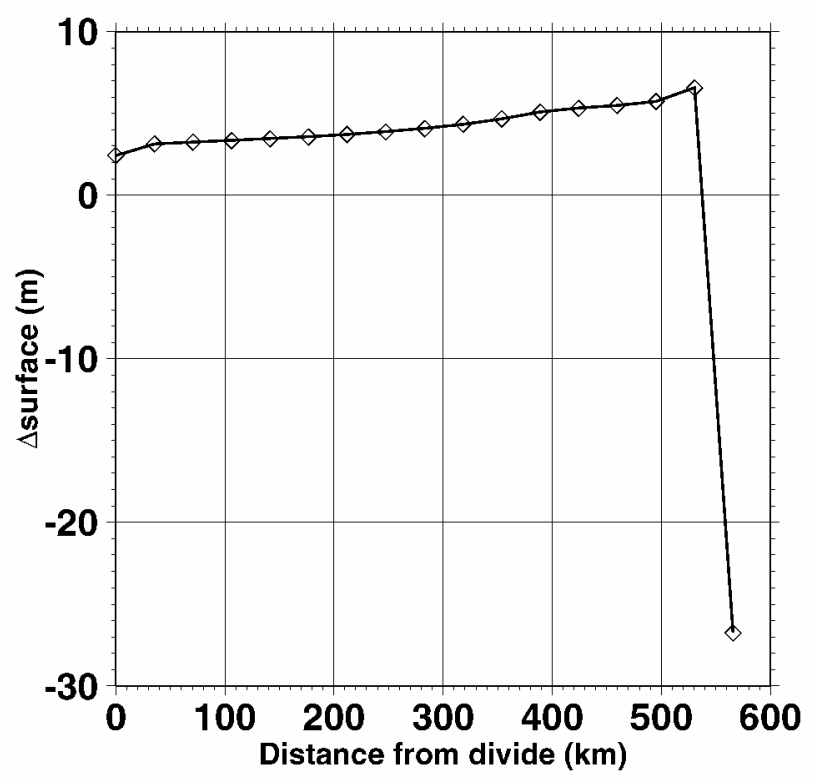

Fig. 1. Steady-state results of the isothermal experiments (U) for difference in surface elevation between the FOA and the corresponding SIA model. The $x$ axis gives the distance from the centre point along the diagonal of the quadratic model domain.

\section{EXPERIMENTS AND RESULTS}

The present work focuses on the effects of the horizontal normal deviatoric stress. The experiments in this work are performed using the framework of the EISMINT model intercomparison experiments (Payne and others, 2000). The quadratic model domain spans $1500 \times 1500 \mathrm{~km}$. The grid resolution is $25 \mathrm{~km}$ in both horizontal coordinate directions (corresponding to $61 \times 61$ grids). Surface mass balance $M_{\mathrm{S}}$ (ice accumulation/ablation rate) and surface temperature $T_{\mathrm{S}}$ are given as a function of the horizontal distance to the summit position alone:

$$
\begin{aligned}
& M_{\mathrm{s}}(x, y) \\
& =\min \left[M_{\max }, S_{\mathrm{b}}\left(R_{\mathrm{el}}-\sqrt{\left(x-x_{0}\right)^{2}+\left(y-y_{0}\right)^{2}}\right)\right], \\
& T_{\mathrm{s}}(x, y)=T_{\min }+S_{\mathrm{T}} \sqrt{\left(x-x_{0}\right)^{2}+\left(y-y_{0}\right)^{2}}
\end{aligned}
$$

where $\left(x_{0}, y_{0}\right)=(0 \mathrm{~km}, 0 \mathrm{~km})$ is the position of the summit, $T_{\min }$ and $S_{\mathrm{T}}$ are the surface temperature at the summit and the radial surface temperature gradient, respectively, and $M_{\text {max }}, S_{\mathrm{b}}$, and $R_{\mathrm{el}}$ are a given upper limit for the accumulation rate, the radial mass-balance gradient and the radial distance of the equilibrium line from the summit, respectively. All the experiments in this work use flat-bed topography as the bottom boundary, and the effects of isostasy are ignored. Basal sliding is also neglected. The steady-state shape of this ideal ice sheet assumes radial symmetry, and the effect of horizontal shear stress is small, so the comparison between steady states of the FOA and SIA models primarily shows the effects of the longitudinal stress gradient.

The enhancement factor is $m=1$ through all the experiments in this work. Parameters $T_{\min }, S_{\mathrm{T}}, S_{\mathrm{b}}, R_{\mathrm{el}}$ and geothermal heat flux $\Gamma$ are set at $238 \mathrm{~K}, 1.67 \times 10^{-2} \mathrm{~K} \mathrm{~km}^{-1}$, $10^{-2} \mathrm{~m} \mathrm{a}^{-1} \mathrm{~km}^{-1}, \quad 450 \mathrm{~km}$ and $42 \mathrm{~mW} \mathrm{~m}^{-2}$, respectively, through all the experiments. All experiments are performed with both the SIA and FOA models. Experiments with the SIA model are labelled with suffix 0, and experiments with the FOA model with 1 . The initial condition is no ice except

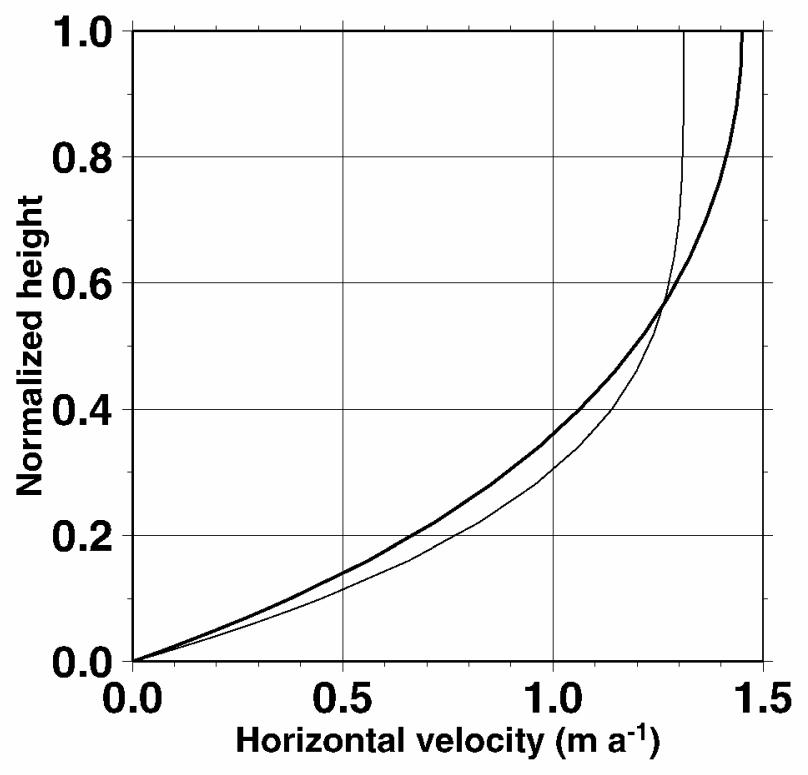

Fig. 2. Vertical profiles of radial velocity (u) at $(12.5 \mathrm{~km}$, $0 \mathrm{~km}$ ) ( half-grid from the divide). The y axis gives the height normalized with ice thickness. Thick and thin lines are the results of $\mathrm{U}_{1}$ and $\mathrm{U}_{0}$, respectively.

where specified. All the experiments are run for at least $200 \mathrm{kyr}$. The time-step is 5 years for dynamics and 20 years for the temperature equation. It takes about a day to finish a 200 kyr calculation using the FOA model on a Hitachi SR8000 supercomputer, and about half an hour using the SIA model.

\subsection{Thermomechanically uncoupled (isothermal) model}

The experiments $U$ are performed using the same boundary conditions as for the corresponding experiment $\mathrm{A}$ in Payne and others (2000); $\bigcup_{1}$ and $U_{0}$ stand for FOA and SIA, respectively. The only difference is that the experiments are performed for $A$ in Equation (2) set constant at $1.0 \times 10^{-16} \mathrm{~Pa}^{-3}$ $\mathrm{a}^{-1}$, such that the computed temperature field does not affect the viscosity of the ice. The parameter $M_{\max }$ is set at $0.5 \mathrm{~m} \mathrm{a}^{-1}$, the same as in experiment A in Payne and others (2000).

Figure 1 shows the difference in the steady-state surface elevations of the $U$ experiment. The effect of the first-order stress on surface elevation (or thickness) is small. Most of the interior parts are thicker by about $10 \mathrm{~m}$ in the FOA (0.1- $0.6 \%$ of the total ice thickness), whereas near the margin the ice is thinner by $20 \mathrm{~m}$ or more $(\sim 4 \%)$. It should be emphasized that the use of a coarse grid for numerical models leads to inaccuracy at some places such as the margin. EISMINTand the present paper serve as a comparison of different numerical methods and may not provide the correct solution. Therefore it cannot be concluded that the FOA is better than the SIA; this paper only discusses the differences between them.

Vertical profiles of flow or stress components near a divide and elsewhere are discussed by Raymond (1983), Reeh (1988) and Dahl-Jensen (1989a,b). Similar results are obtained in this work. Figure 2 shows the vertical profiles of the horizontal velocity component at half a grid distance away from the ice divide (note that staggered grids are used for calculation of the horizontal velocity) by experiments $U_{1}$ and $U_{0}$. The horizontal velocity of $U_{1}$ is larger than that of $U_{0}$ 


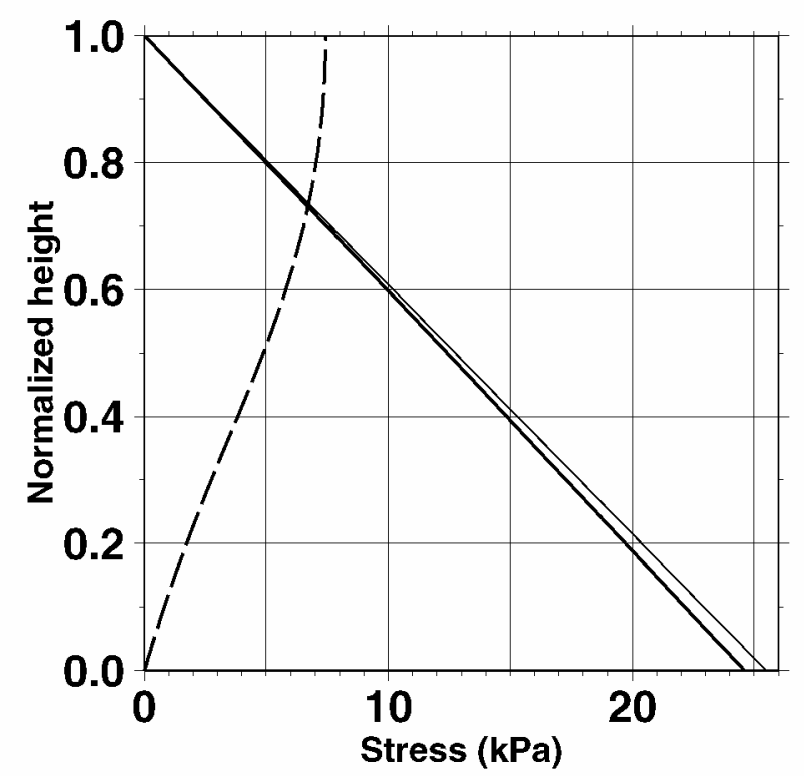

Fig. 3. Vertical profiles of stress components $\left(\sigma_{x z}, \sigma_{x x}^{\prime}\right)$ at $(25 \mathrm{~km}, 0 \mathrm{~km})$. The $y$ axis gives the height normalized with ice thickness. Thick and thin lines are the result of $\mathrm{U}_{1}$ and $\mathrm{U}_{0}$, respectively. Solid and dashed lines for each thickness are $\sigma_{x z}$ and $\sigma_{x x}^{\prime}$ respectively. Note that for $\bigcup_{0}, \sigma_{x x}^{\prime}$ is neglected and set to 0 .

near the surface, and smaller near the bed. However, the horizontal ice flux of the steady-state solutions at the same point must be equal in both experiments, because the boundary condition of surface mass balance in the present work is explicitly a function of the distance from the ice divide, independent of thickness. The smaller velocity of $\bigcup_{1}$ at deeper layers dominates the effect of the faster upper layers, so the vertically integrated velocity of $U_{1}$ is smaller than that of $U_{0}$. As a result, the FOA ice thickness is larger than the SIA ice thickness. The difference between the two experiments at one grid further away from the ice divide is similar to, but smaller than, that at the first grid.

The difference in vertical profiles of horizontal velocity

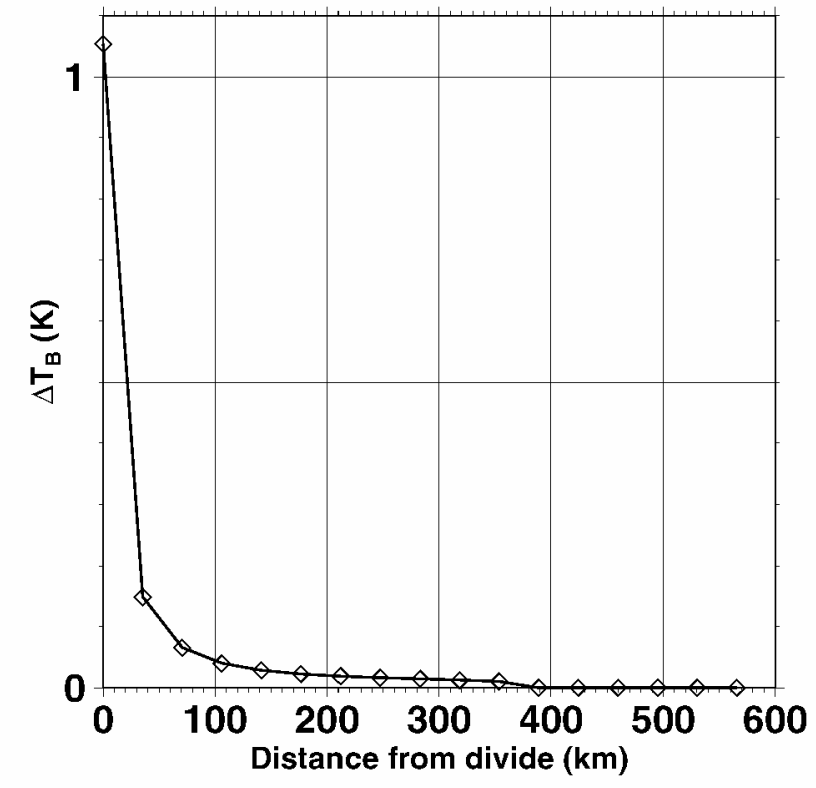

Fig. 4. Steady-state results of the isothermal experiments (U) for difference in basal temperatures between the FOA and the SIA model. The $x$ axis gives the distance from the centre point along the diagonal of the quadratic model domain.

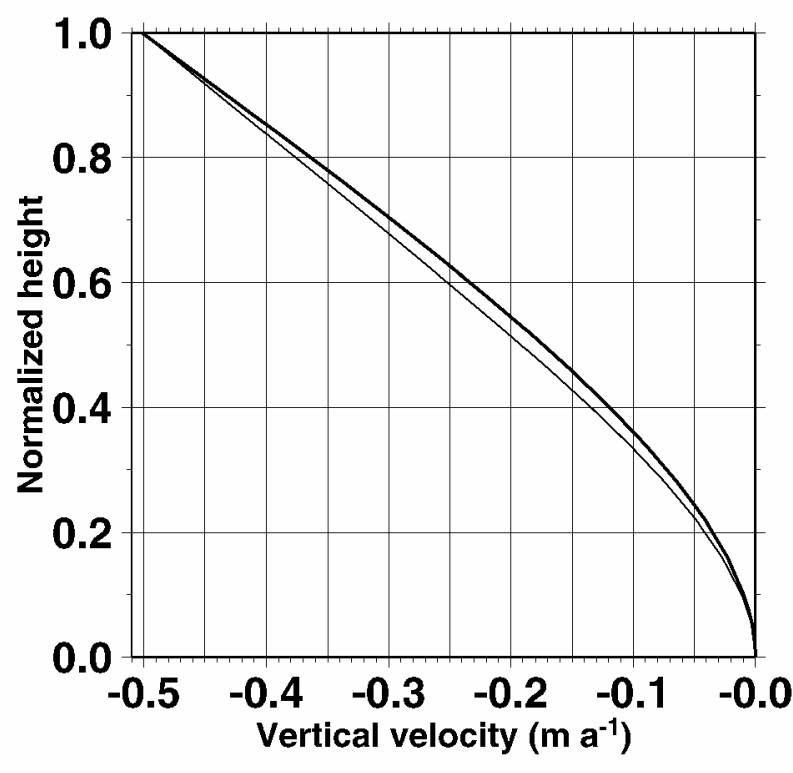

Fig. 5. Vertical profiles of vertical velocity $(w)$ below the divide. The y axis gives the height normalized with ice thickness. Thick and thin lines are the results of $\mathrm{U}_{1}$ and $\mathrm{U}_{0}$, respectively.

between the two models can be explained qualitatively in terms of stress profiles. Figure 3 shows the vertical profile of stress components one grid away from the ice divide. As discussed by Raymond (1983), Reeh (1988) and Dahl-Jensen (1989a, b), this profile is qualitatively similar for the entire interior region. The FOA force-balance Equation (la) in two dimensions becomes

$$
\frac{\partial \sigma_{x z}}{\partial z}=\rho_{\mathrm{I}} g \frac{\partial h}{\partial x}-\left(2 \frac{\partial \sigma_{x x}^{\prime}}{\partial x}\right) .
$$

In the SIA, the second term on the righthand side is neglected. Near the divide where the surface gradient is negative, the gradient of longitudinal deviatoric stress is positive (divergence flow). Thus,

$$
\left.\frac{\partial \sigma_{x z}}{\partial z}\right|^{\mathrm{SIA}}<\left.\frac{\partial \sigma_{x z}}{\partial z}\right|^{\mathrm{FOA}},
$$

which corresponds to Figure 3. The constitutive Equation (2c) in two dimensions is

$$
\frac{\partial u}{\partial z}=2 m A(T)\left[\left(\sigma_{x z}\right)^{2}+\left(\sigma_{x x}^{\prime}\right)^{2}\right]^{(n-1) / 2} \sigma_{x z} .
$$

At the bed, where $\sigma_{x x}^{\prime}=0$, Equation (14) becomes

$$
\frac{\partial u}{\partial z} \propto\left(\sigma_{x z}\right)^{n}
$$

which is equivalent to the SIA formulation. At the bed, the horizontal velocity in the FOA is smaller than in the SIA (Fig. 2), because $\sigma_{x z}$ of $U_{1}$ is smaller than that of $U_{0}$ at the bed.

Near the surface, where $\sigma_{x z} \rightarrow 0$, Equation (14) becomes

$$
\frac{\partial u}{\partial z} \propto\left(\sigma_{x x}^{\prime}\right)^{n-1} \sigma_{x z}
$$

Since the SIA neglects the $\sigma_{x x}^{\prime}$ term in Equation (16), $\partial u / \partial z$ approaches zero faster in the SIA than in the FOA. Thus, near the surface, the vertical profile of horizontal velocity is steeper in the SIA than in the FOA (Fig. 2).

In spite of minor differences in surface elevation, basal temperatures differ substantially near the divide. Figure 4 shows the difference in basal temperatures (in terms of temperature below local pressure-melting point) between the 


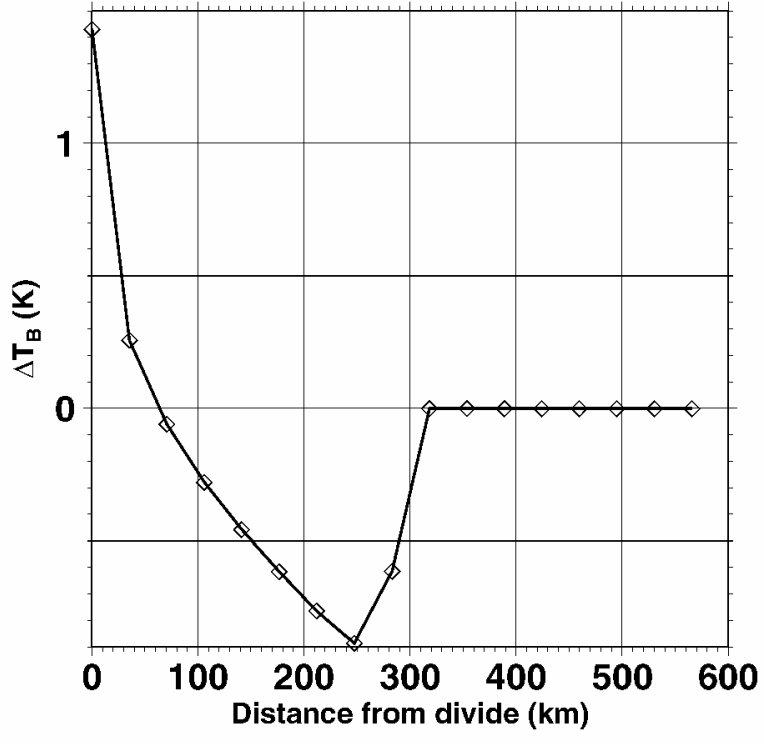

Fig. 6. Steady-state results of experiment $\mathrm{A}$ for difference in basal temperature between result of $A_{1}$ and that of $A_{0}$. The $x$ axis gives the distance from the centre point along the diagonal of the quadratic model domain.

two experiments. Inclusion of the first-order stress gradients produces a warmer base below the summit by $1 \mathrm{~K}$ because of the differences in vertical advection (Fig. 5). Note that the result shows clearly that a "hot spot" (Dahl-Jensen, 1989a) below the divide is produced by the first-order model.

In these experiments, thermomechanical coupling is not performed, and the temperature differences do not affect the softness of ice. If the flow parameter $A$ is made temperature-dependent, warmer ice than in the above experiments may cause higher velocities, and the ice may become thinner. In section 3.2, the effects of thermodynamic coupling are discussed.

\subsection{EISMINT experiments}

In this subsection, $A$ series experiments are performed using the same boundary conditions as for the corresponding EISMINT experiments A in Payne and others (2000). Now thermomechanical coupling is applied. It may be concluded that the effects of inclusion of the first-order stress gradients do not change much in comparison to the isothermal experiments.

Figure 6 shows the difference in basal temperatures between the steady-state results of $A_{1}$ and $A_{0}$ along the diagonal section. It can be divided into three regions based on the different effects of the first-order stress gradients: (1) basal temperatures of $A_{1}$ are higher near the ice divide; (2) they are lower away from the divide $(50<d \leq 300 \mathrm{~km})$; and (3) they are equal in the region at the pressure-melting point $(300 \mathrm{~km}<d)$. The behaviour near the ice divide can be explained by the difference in the vertical advection term, as in the U series ("hot spot").

The behaviour away from the divide is more complex. In this case, the difference in strain heating between $A_{1}$ and $A_{0}$ is large compared to the difference in strain heating of experiments $U$, though the effect of first-order stress gradients is qualitatively the same. The reason for this large difference in basal strain heating seems to be positive feedback of temperature and strain, which occurs only in thermomechanical coupling: a decrease of strain heating causes a decrease in

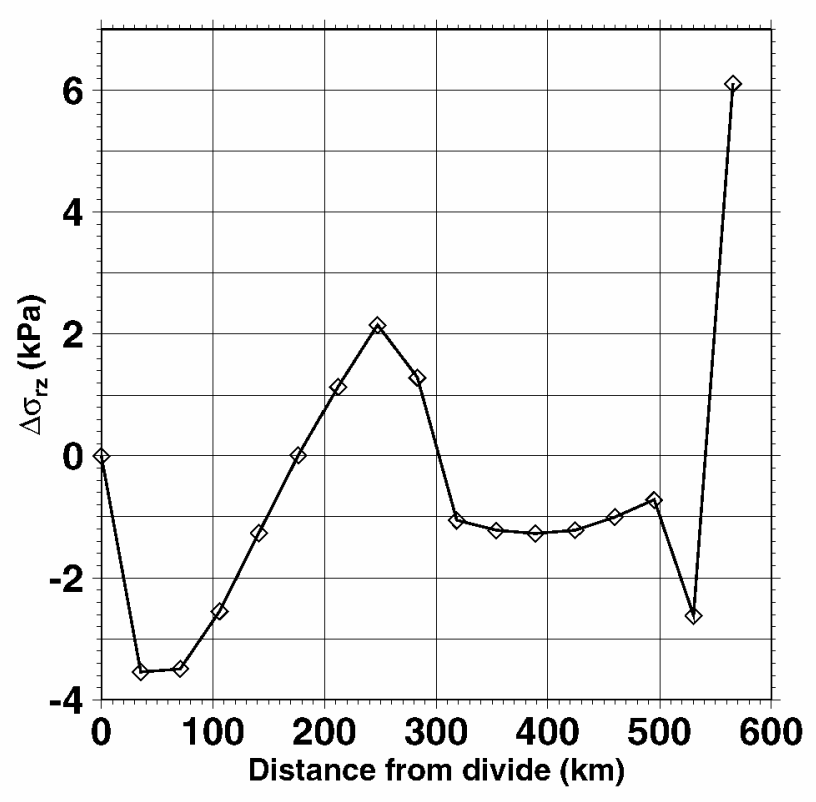

Fig. 7. Difference in basal vertical shear stress between steadystate solutions of experiments $\mathrm{A}_{1}$ and $\mathrm{A}_{0}$. The $x$ axis gives the distance from the centre point along the diagonal of the quadratic model domain.

temperature, which in turn leads to harder ice. The harder ice further leads to smaller strain rates, which further reduce strain heating. There is a slight difference for regions with basal pressure melting. The melting region of $A_{1}$ is narrower by about $30 \mathrm{~km}$.

For some regions, the large difference in basal strain heating has a more complicated explanation. It derives mainly from the difference in basal shear stress in the two models. Figure 7 shows the difference in basal shear stress components between $A_{1}$ and $A_{0}$. Except for the region near the area of the pressure-melting point $(0<d \lesssim 150 \mathrm{~km})$, the basal shear stress of $A_{1}$ is smaller than that of $A_{0}$. Since strain heating is the product of stress and strain rate, strain heating is reduced in this region. However, the region is characterized by higher shear stress as well as lower strain heating. The temperature is low enough that shear strain rate becomes small enough to cancel the effect of higher shear stress for strain heating. Further detailed analysis is required to clarify the feedback loop of thermomechanical coupling in this area. It may be due to the (small) difference in temperature advection from upper layers.

The steady-state surface elevation is affected by a temperature difference. However, the effect of the first-order stress gradients on the steady-state shape is small. Figure 8 shows the difference in surface elevation between $A_{1}$ and $A_{0}$. The effect of the first-order stress on surface elevation is generally the same as in the isothermal experiment $U$. There is a slightly different behaviour near the divide, where the surface elevation is lower in the FOA than in the SIA, due to thermomechanical coupling (see section 3.1). Warmer ice near the divide results in softer ice, ice flow becomes faster, and consequently the ice sheet becomes thinner.

\subsection{Diagnosis of age profiles below a steady-state summit}

The vertical age profile at the ice divide (horizontal velocity $=0$ ) of the steady-state ice sheet is computed by integrating 


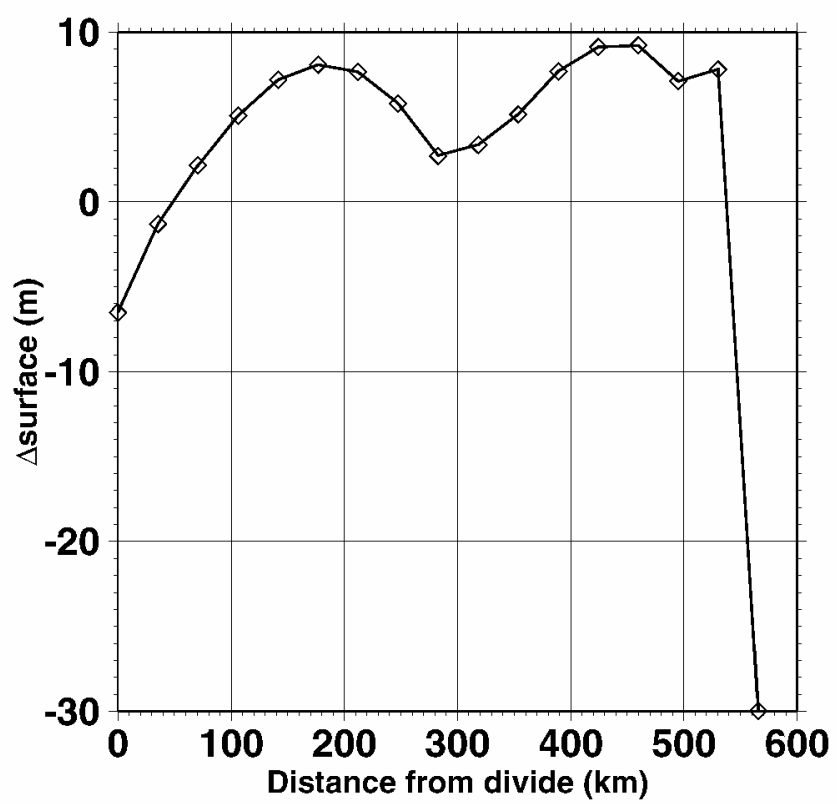

Fig. 8. Steady-state results for difference in surface elevation between $\mathrm{A}_{1}$ and $\mathrm{A}_{0}$. The horizontal axis gives the distance from the centre point along the diagonal of the quadratic model domain.

the reciprocal of the vertical velocity component along the vertical axis to the surface:

$$
\mathcal{A}=\int_{z}^{h} \frac{\mathrm{d} z}{w} .
$$

In a steady state, the downward distance an ice particle moves in 1 year is equal to vertical velocity, $w$; thus Equation (17) is obtained. It is assumed that the surface mass balance remains unchanged throughout the time integration. The region near the bed, where vertical velocity approaches zero (its reciprocal becomes infinite), is not considered. In this subsection, $\mathbf{Z}$ series experiments are performed using the same boundary conditions as in the corresponding experiments A in Payne and others (2000) except that the accumulation is reduced to $10 \%$ of the accumulation in experiments $A$.

Figure 9 shows the results of age calculations at the ice divide of experiments $A$ and $Z$. The differences gradually increase toward deeper parts. The accumulation of experiment $A$ is $50 \mathrm{~cm} \mathrm{a}^{-1}$, a typical value for Greenland drilling sites, and the accumulation of experiment $Z$ is $5 \mathrm{~cm} \mathrm{a}^{-1}$, a typical value for Antarctic drilling sites. At $2000 \mathrm{~m}$ depth, the age of the ice in experiment $Z_{1}$ is $130 \mathrm{kyr}$, while in $Z_{0}$ is $120 \mathrm{kyr}$. The difference is as much as $10 \mathrm{kyr}(\sim 10 \%$ of age). At $3000 \mathrm{~m}$ depth, the age of the ice in experiment $A_{1}$ is $18 \mathrm{kyr}$, while that in experiment $A_{0}$ is $16 \mathrm{kyr}$. The difference is as much as $2 \mathrm{kyr}$ $(\sim 10 \%)$. In this case, the vertical velocity components at both surface and base boundaries must be the same for both approximations because of the prescribed boundary conditions. The largest difference occurs at middle depth where the vertical velocity in the FOA is slower than that in the SIA at the same depth (Fig. 5), as previously discussed in Raymond (1983), Reeh (1988) and Dahl-Jensen (1989a).

This is the case for such an idealized configuration. In the case of realistic ice sheets with surface elevation, velocity profiles and surface accumulation changing through time, the difference in the calculated age of the ice may be considerably larger.
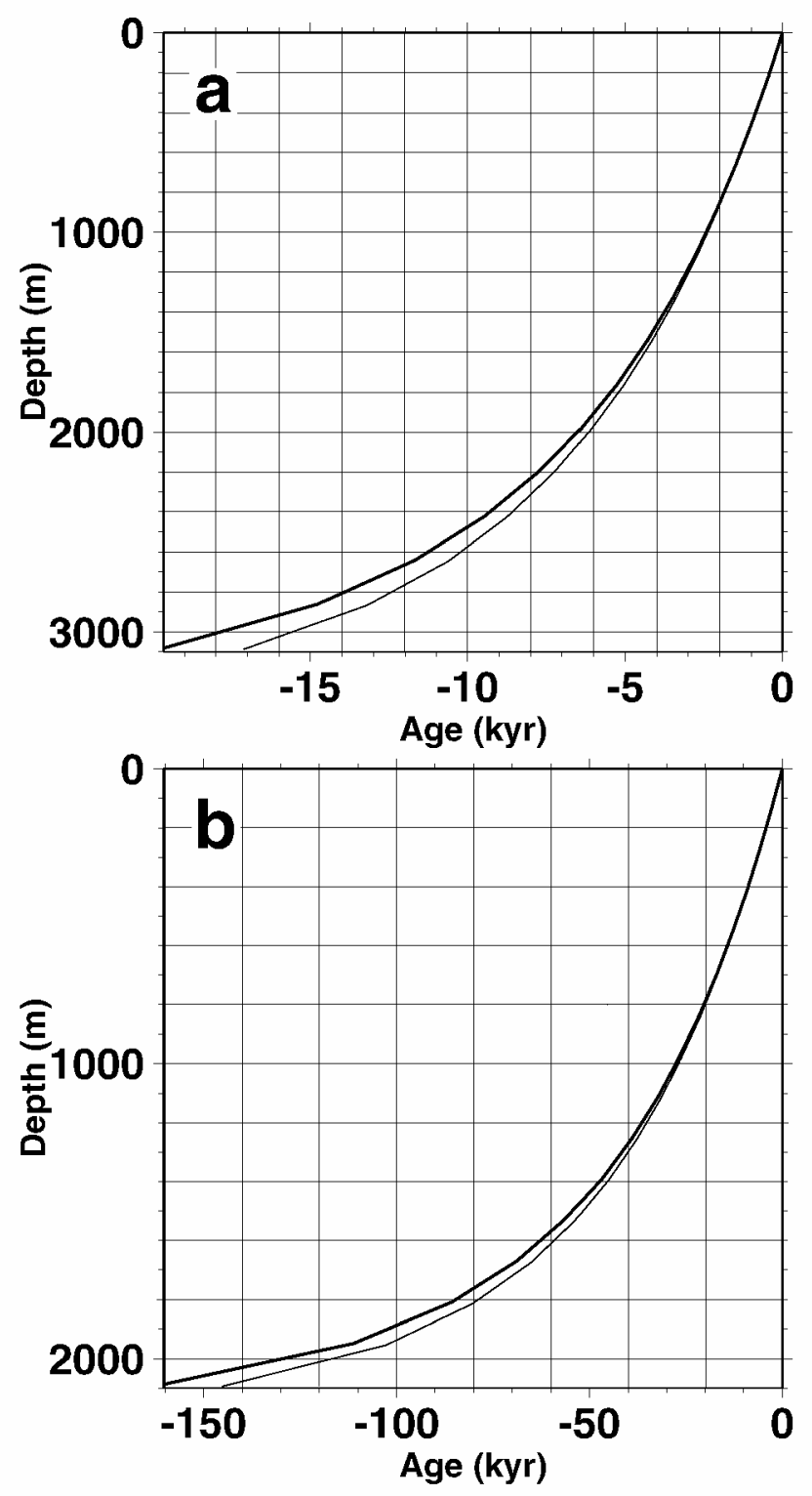

Fig. 9. Vertical profiles of age of the ice below the ice divide of experiments $\mathbf{A}(a)$ and $\mathbf{Z}(b)$. The y axis gives the depth below the ice surface. Thick and thin lines are the results of the FOA and SIA models, respectively.

\section{CONGLUSION AND PROSPEGTS}

In the present work, a three-dimensional numerical icesheet model with thermodynamic coupling including the first-order mechanics is presented. The model is applied to an idealized ice sheet with radial symmetry to investigate the effects of the normal deviatoric-stress gradients on ice thickness, basal temperature and age distribution. The main conclusions of the present work are as follows:

The difference in age profiles below the steady-state summit between the FOA and the SIA reaches as much as $10 \%$ of total age near the base.

Basal temperature differs as much as $\pm 1 \mathrm{~K}$ between the FOA and the SIA, due either to the difference in the vertical advection (near the divide) or to the difference in strain heating (away from the divide).

The difference in surface elevation of steady states between the FOA and the SIA models is generally small (only a few tens of metres).

The insignificant differences between the results obtained 
by the FOA and SIA models are due to the simple configuration of the experiments. Because of the flat bed and the radial symmetry, the role of higher-order stress components is expected to be small in almost the whole domain. The implications of the present work are limited, and it cannot be concluded from the present result alone that the FOA model is better than the SIA model. However, careful interpretation of the simulated flow and geometry of ice sheets is required in certain cases, because of the different characteristics of the two models.

In addition to the idealized study presented, several applications of the first-order model developed in the present work are planned for the future. Firstly, the presented FOA model can be used for dating. Secondly, it can be an effective tool to connect ice microphysics to large-scale ice-sheet behaviour. Thirdly, it can be used to study ice-stream dynamics. In addition, basal sliding, which is also important for ice-stream dynamics, may present different behaviour in the two approximations, due to the difference between the basal shear traction, used in FOA models, and the driving stress, used in SIA models, to parameterize sliding. Therefore, studies on the stability of marine ice sheets such as the West Antarctic ice sheet require the application of the FOA model; this is a planned future application of the presented model.

\section{AGKNOWLEDGEMENTS}

The authors wish to thank L.W. Morland, the editor, and two anonymous reviewers for their useful comments.

\section{REFERENGES}

Abe-Ouchi, A. 1993. Ice sheet response to climate changes: a modelling approach. Zürcher Geogr. Schr. 54.

Azuma, N. 1994. A flow law for anisotropic ice and its application to ice sheets. Earth Planet. Sci. Lett., 128(3-4), 601-614.

Azuma, N. and K. Goto-Azuma. 1996. An anisotropic flow law for ice-sheet ice and its implications. Ann. Glaciol., 23, 202-208.

Azuma, N. and 6 others. 1999. Textures and fabrics in the Dome F (Antarctica) ice core. Ann. Glaciol., 29, 163-168.

Baral, D., K. Hutter and R. Greve. 2001. Asymptotic theories of large-scale motion, temperature and moisture distribution in land-based polythermal ice sheets: a critical review and new developments. Appl. Mech. Rev., 54(3), 215-256.
Blatter, H. 1995. Velocity and stress fields in grounded glaciers: a simple algorithm for including deviatoric stress gradients. F. Glaciol., 41(138), 333-344.

Budd, W. F., B. Coutts and R. C. Warner. 1998. Modelling the Antarctic and Northern Hemisphere ice-sheet changes with global climate through the glacial cycle. Ann. Glaciol., 27, 153-160.

Colinge, J. and H. Blatter. 1998. Stress and velocity fields in glaciers: Part I Finite-difference schemes for higher-order glacier models. 7. Glaciol., 44(148), 448-456.

Dahl-Jensen, D. 1989a. Steady thermomechanical flow along two-dimensional flow lines in large grounded ice sheets. 7. Geophys. Res., 94(B8), $10,355-10,362$

Dahl-Jensen, D. 1989b. Two-dimensional thermo-mechanical modelling of flow and depth-age profiles near the ice divide in central Greenland. Ann. Glaciol., 12, 31-36.

Greve, R. 1997. A continuum-mechanical formulation for shallow polythermal ice sheets. Philos. Trans. R. Soc. London, Ser. A, 355(1726), 921-974.

Greve, R., M. Weis and K. Hutter. 1998. Palaeoclimatic evolution and present conditions of the Greenland ice sheet in the vicinity of Summit: an approach by large-scale modelling. Palaeoclimates, 2(2-3), 133-161.

Hondoh, T. and 15 others. 1999. Basic analyses of Dome Fuji deep ice core. Part 2: physical properties. Polar Meteorol. Glaciol., 13, 90-98.

Huybrechts, P. 1990. A 3-D model for the Antarctic ice sheet: a sensitivity study on the glacial-interglacialcontrast. Climate Dyn., 5(2), 79-92.

Huybrechts, P. 1992. The Antarctic ice sheet and environmental change: a three-dimensional modelling study. Ber. Polarforsch. 99.

Marshall, S. J., L. Tarasov, G. K. C. Clarke and W. R. Peltier. 2000. Glaciological reconstruction of the Laurentide ice sheet: physical processes and modelling changes. Can. 7. Earth Sci., 37(5), 769-793.

Miyamoto, A. and 9 others. 1999. Ice-sheet flow conditions deduced from mechanical tests of ice core. Ann. Glaciol., 29, 179-183.

Paterson, W. S. B. 1994. The physics of glaciers. Third edition. Oxford, etc., Elsevier.

Pattyn, F. 1996. Numerical modelling of a fast-flowing outlet glacier: experiments with different basal conditions. Ann. Glaciol., 23, 237-246.

Pattyn, F. 2000. Ice-sheet modelling at different spatial resolutions: focus on the grounding zone. Ann. Glaciol., 31, 211-216.

Pattyn, F. and H. Decleir. 1998. The Shirase flow-line model: an additional tool for interpreting the Dome-Fuji signal. Polar Meteorol. Glaciol., 12, 104-111.

Payne, A. J. 1999. A thermomechanical model of ice flow in West Antarctica. Climate Dyn., 15(2), 115-125.

Payne, A. J. and 10 others. 2000. Results from the EISMINT model intercomparison: the effects of thermomechanical coupling. F. Glaciol., 46(153), 227-238.

Raymond, C. F. 1983. Deformation in the vicinity of ice divides. F. Glaciol., 29(103), 357-373

Reeh, N. 1988. A flow-line model for calculating the surface profile and the velocity, strain-rate, and stress fields in an ice sheet. F. Glaciol., 34(116), 46-54.

Ritz, C., A. Fabre and A. Letréguilly. 1997. Sensitivity of a Greenland icesheet model to ice flow and ablation parameters: consequences for the evolution through the last glacial cycle. Climate Dyn., 13(1), 11-24. 